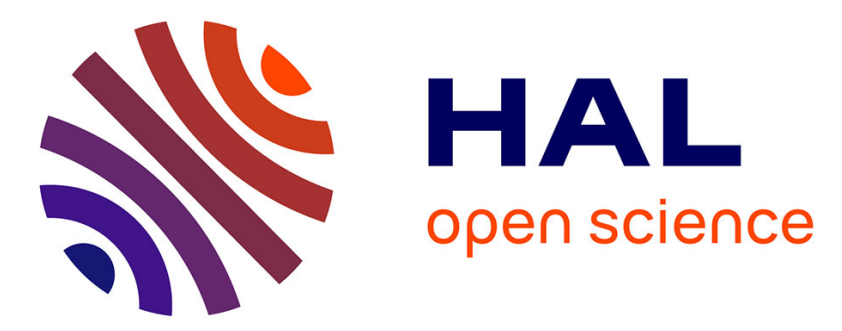

\title{
Constrained least squares detector for OFDM/SDMA-based wireless networks
}

S. Thoen, Luc Deneire, L. van Der Perre, M.G.E. Engels, H. de Man

\section{To cite this version:}

S. Thoen, Luc Deneire, L. van Der Perre, M.G.E. Engels, H. de Man. Constrained least squares detector for OFDM/SDMA-based wireless networks. IEEE Transactions on Wireless Communications, 2003, 2 (1), pp.129-140. 10.1109/TWC.2002.806377 . hal-00187776

\section{HAL Id: hal-00187776 https://hal.science/hal-00187776}

Submitted on 15 Nov 2007

HAL is a multi-disciplinary open access archive for the deposit and dissemination of scientific research documents, whether they are published or not. The documents may come from teaching and research institutions in France or abroad, or from public or private research centers.
L'archive ouverte pluridisciplinaire HAL, est destinée au dépôt et à la diffusion de documents scientifiques de niveau recherche, publiés ou non, émanant des établissements d'enseignement et de recherche français ou étrangers, des laboratoires publics ou privés. 


\title{
Constrained Least Squares Detector for OFDM/SDMA-Based Wireless Networks
}

\author{
Steven Thoen, Student Member, IEEE, Luc Deneire, Member, IEEE, Liesbet Van der Perre, \\ Marc Engels, Member, IEEE, and Hugo De Man, Fellow, IEEE
}

\begin{abstract}
The two major obstacles toward high-capacity indoor wireless networks are distortion due to the indoor channel and the limited bandwidth which necessitates a high spectral efficiency. A combined orthogonal frequency division multiplexing (OFDM) / spatial division multiple access (SDMA) approach can efficiently tackle both obstacles and paves the way for cheap, high-capacity wireless indoor networks [27], [26]. The channel distortion due to multipath propagation is efficiently mitigated with OFDM while the bandwidth efficiency can be increased with the use of SDMA. However, to keep the cost of an indoor wireless network comparable to its wired counterpart's cost, low-complexity SDMA processors with good performance are of special interest. In this paper, we propose a new multiuser SDMA detector which is designed for constant modulus signals. This constrained least squares (CLS) receiver, which deterministically exploits the constant modulus nature of the subcarrier modulation to achieve better separation, is compared in terms of performance and complexity with the zero forcing (ZF) and the minimum mean square error (MMSE) receiver. Additionally, since the CLS detector relies on reliable channel knowledge at the receiver, we propose a strategy for estimating the multiple input multiple output (MIMO) channels. Simulations for a Hiperlan II-based [13] case-study show that the CLS detector significantly outperforms the $\mathrm{ZF}$ detector and comes close to the performance of the MMSE detector for QPSK. For higher order M-PSK, the CLS detector outperforms the MMSE detector. Furthermore, the estimation complexity for the CLS detector is substantially lower than that for the MMSE detector which additionally requires estimation of the noise power.
\end{abstract}

Index Terms-Adaptive antennas, antenna diversity, channel estimation, orthogonal frequency division multiplexing (OFDM), space division multiple access (SDMA).

\section{INTRODUCTION}

W IRELESS local access networks (WLANs) provide user mobility and inexpensive network installation and reconfiguration compared to their wired counterparts. However, in order to be competitive with wired LANs, future WLANs should offer both high capacity and low cost. The design of such high-capacity low-cost wireless networks is complicated by spectral limitations and distortion due to the indoor channel.

First, orthogonal frequency division multiplexing (OFDM) provides an elegant solution to the distortion problem. The indoor channel features multipath propagation, characterized by

Manuscript received February 26, 2001; revised September 4, 2001; accepted January 16,2002. The editor coordinating the review of this paper and approving it for publication is C. Tellambura.

The authors are with Resonext Communications, Technologielaan 4, 3001 Belgium and with IMEC, Katholieke Universiteit Leuven, Leuven 3001, Belgium (e-mail: steven.thoens@ resonext.com).

Digital Object Identifier 10.1109/TWC.2002.806377 its delay spread. Due to the envisaged high data rates, the multiple paths give rise to intersymbol interference (ISI), which distorts the signal and results in a frequency-selective channel response. OFDM modulation with cyclic prefix insertion mitigates ISI by extending the symbol period as the data is multiplexed on orthogonal subcarriers [2]. As such, it converts a frequency selective channel into a number of parallel flat fading channels which can be easily equalized with simple one-tap equalizers. Furthermore, the (de)modulation can be executed efficiently via the fast Fourier transform (FFT). Hence, OFDM is the preferred modulation type for high-capacity WLAN applications and has been adopted in WLAN standards such as IEEE 802.11a and ETSI Hiperlan II [13].

Second, SDMA provides high bandwidth efficiency which is crucial because of spectral limitations. It is well known that the capacity of a wireless system can be increased dramatically by employing multiple antennas [8], [23], [29]. We propose to reuse the bandwidth within one cell by spatial division multiple access (SDMA) [26], [27] at the basestation. Due to the wide angular spread of the received signals in indoor environments, we cannot rely on beamforming and wideband SDMA is needed. Essentially, SDMA separates multiple simultaneously transmitting users based on their different spatial signatures by processing the signals received at an antenna array.

Finally, a combined OFDM/SDMA approach can benefit from the advantages of both OFDM and SDMA to enable a high-capacity WLAN. Furthermore, the inherent parallelism of OFDM allows per-subcarrier SDMA processing, resulting in a considerably lower implementation cost compared to single-carrier SDMA. A vast number of possible multiuser detection algorithms has originated in the CDMA context [28], all of which can be applied to solve the individual SDMA problems on the subcarriers. For a cost-efficient WLAN, we are interested in low-complexity multiuser detectors that exhibit good performance.

In this paper, we derive a new detector which is designed specifically for constant modulus signals and hence is applicable to M-PSK signals. The constant modulus property of transmitted symbols historically has been exploited first via the Constant Modulus Algorithm proposed by Godard [10]. This algorithm has been applied extensively in various forms and scenarios for achieving blind beamforming [1] and equalization (see [14] and references therein). An analytical constant modulus algorithm has been proposed in [25]. In this paper however, the constant modulus property is exploited deterministically and channel knowledge is assumed to be available at the receiver side. The proposed constrained least squares (CLS) detection algorithm, which essentially is a linear 


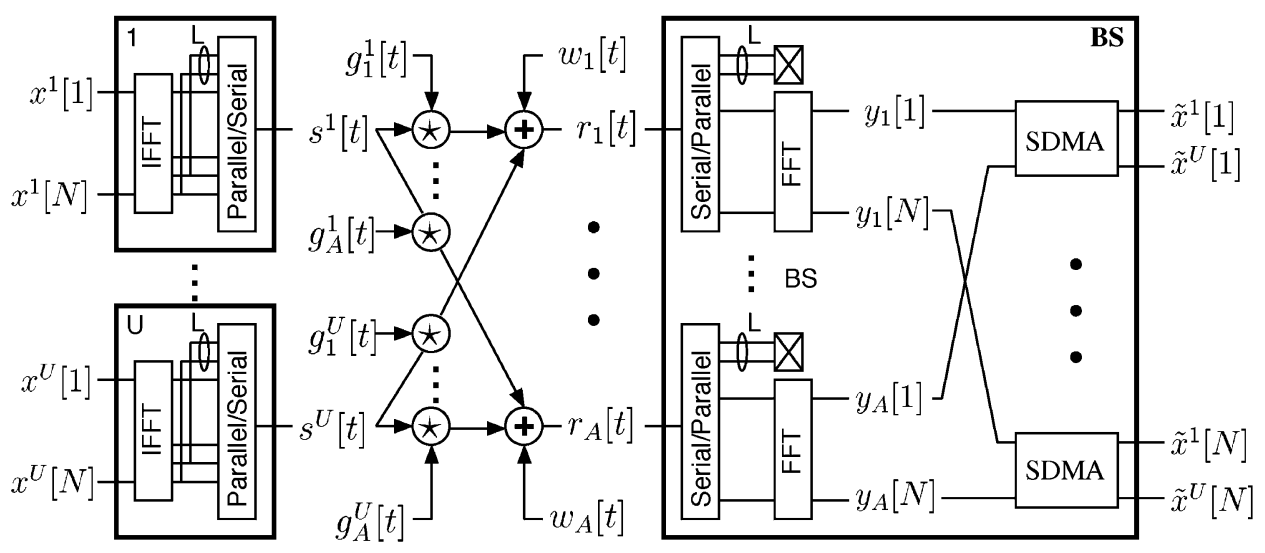

Fig. 1. System model for OFDM/SDMA in the uplink.

detector, is analyzed in terms of performance and complexity. The proposed algorithm is benchmarked against two other well-known receivers, namely the zero forcing (ZF) and the minimum mean square error (MMSE) detector [28], since they also belong to the class of linear receivers. We show that the CLS detector, by explicitly taking the constant modulus character of the signals into account, can construct an optimal linear transformation which for our case study outperforms the ZF and comes close to (and for higher order constellations outperforms) the MMSE linear transformations in terms of average bit error rate (BER).

Since the CLS detector relies on knowledge of the channel propagation characteristics of the simultaneously transmitting users, these parameters need to be estimated in practical systems. Therefore, we propose a low complexity pilot-based multiple-input multiple-output (MIMO) channel estimator which can be designed to tradeoff pilot overhead and complexity with estimation accuracy. With this estimator, accurate estimations can be achieved such that the performance degradation of the SDMA detectors due to nonideal channel knowledge is small.

The proposed CLS detector has the additional advantage that the estimation complexity is substantially lower than for the MMSE detector, which also requires estimation of the average noise power. Since the estimation complexity is an order of magnitude higher than the processing complexity, the gain in the overall basestation complexity is shown to be substantial.

This paper is organized as follows. In Section II, the system model is defined. Section III derives the proposed Constrained Least Squares detector. Section IV describes the proposed channel and noise power estimation strategy. Section V analyzes the performance of the detection algorithms both with perfect channel knowledge and in combination with channel parameter estimation. Finally, some conclusions are drawn in Section VI.

\section{SYSTEM MODEL}

\section{A. Notations}

In this paper, normal letters indicate scalar quantities, boldface letters represent vectors, and boldface capitals indicate matrices. Furthermore, $\mathbf{X}^{T}$ denotes the transpose of a matrix $\mathbf{X}$ while $\mathbf{X}^{H}$ denotes its conjugate transpose.

\section{B. OFDM/SDMA System Setup}

The proposed OFDM/SDMA system consists of several user terminals and one basestation $B S$. Each of the users has a single antenna in order to keep the cost of the user terminals low, while the basestation has an array of $A$ antennas. In each frame, $U$ users are assigned which can simultaneously transmit OFDM modulated symbols to the basestation. The basestation then separates the streams of the $U$ users by processing the signal vectors received at its antenna array, resulting in a $U$-fold bandwidth reuse.

The system under consideration is schematized in Fig. 1 which represents the $U$ simultaneous users and the basestation $B S$. Each user $u$ feeds its data $x^{u}[n]$ in blocks of $N$ symbols into an $N$-tap inverse FFT (IFFT) operator to obtain the time-domain sequence $s^{u}[t]$. A cyclic extension of length $L$ is inserted into the sequence, which is then converted to a serial stream. The resulting sequence $\left[s^{u}[N-L+1] \cdots s^{u}[N] s^{u}[1] \cdots s^{u}[N]\right]$ is transmitted through the $A$ convolutional channels $g_{a}^{u}[t]$, with $g_{a}^{u}[t]$ the baseband representation of the multipath channel from user $u$ to antenna $a$. At the basestation, each antenna $a$ receives the convolutional mixture $r_{a}^{u}[t]=\sum_{u=1}^{U} g_{a}^{u}[t] \star s^{u}[t]$ and an AWGN term $w_{a}[t]$. Subsequently, the operations of the transmitter are inverted. After discarding the cyclic prefix and taking the $N$-tap FFT of each received signal, we end up with $A$ received sequences $y_{a}[n]$ on each subcarrier. These signals $y_{a}[n]$ are then postprocessed by the basestation to separate the distinct users and to provide estimates $\tilde{x}^{u}[n]$ for the transmitted symbols $x^{u}[n]$.

If $L$ is larger than the channel length and the users are properly symbol synchronized, ${ }^{1}$ the linear channel convolution is observed as cyclic by the basestation. Thus, in the frequency domain it becomes equivalent to multiplication with the discrete Fourier transform of the channel, $h_{a}^{u}[n]$. Then, we can write for each subcarrier $n$

$\underbrace{\left[\begin{array}{c}y_{1}[n] \\ \vdots \\ y_{A}[n]\end{array}\right]}_{\mathbf{y}[n]}=\underbrace{\left[\begin{array}{ccc}h_{1}^{1}[n] & \cdots & h_{1}^{U}[n] \\ \vdots & \ddots & \vdots \\ h_{A}^{1}[n] & \cdots & h_{A}^{U}[n]\end{array}\right]}_{\mathbf{H}[n]} \cdot \underbrace{\left[\begin{array}{c}x^{1}[n] \\ \vdots \\ x^{U}[n]\end{array}\right]}_{\mathbf{x}[n]}+\underbrace{\left[\begin{array}{c}n_{1}[n] \\ \vdots \\ n_{A}[n]\end{array}\right]}_{\mathbf{n}[n]}$.

${ }^{1}$ Note that the cyclic prefix length can be designed robustly to provide synchronization margin. 
TABLE I

WLAN CASE-STUDY SPECIFICATION

\begin{tabular}{c|c}
\hline Network capacity & 48 Msymbols/s \\
User signaling rate & 12 Msymbols/s \\
Number of subcarriers & $N=64$ \\
Number of data subcarriers & $N_{d}=48$ \\
Cyclic prefix length & $L=16$ \\
Number of simultaneous users & $U \leq 4$ \\
Number of antennas & $A=4$ \\
\hline
\end{tabular}

Clearly, due to the OFDM modulation, the SDMA problem falls apart in multiple parallel SDMA problems which can be solved independently per subcarrier, as depicted in Fig. 1. Hence, the SDMA processing is transformed into $N$ SDMA processors running $N+L$ times slower than the time-domain symbol rate. A generic SDMA processor calculates estimates $\tilde{x}^{u}[n]$ for the transmitted symbols $x^{u}[n]$, using the received $y_{a}[n]$ and an estimate $\tilde{\mathbf{H}}[n]$ of the channel coefficients matrix $\mathbf{H}[n]$. In this paper, we derive a new SDMA processor, namely the CLS detector where the estimates $\tilde{x}^{u}[n]$ are constrained to be constant envelope, and assess its performance. Furthermore, since the performance of any SDMA detector depends heavily on the precision of its channel estimate, we developed a MIMO channel estimator. This estimator, which is described in Section IV, allows accurate estimation of the channels of all $U$ users for all $A$ antennas based on OFDM training symbols transmitted at the start of a signaling frame.

\section{OFDM/SDMA Case Study}

For analyzing the performance and complexity of the proposed CLS receiver and the MIMO channel estimator, we utilize a specific case study which consists of a basestation equipped with four antennas and up to four simultaneously transmitting mobile users. The OFDM-based ETSI Hiperlan II indoor wireless LAN standard [13] is used for all physical layer parameters. Each user signals at $12 \mathrm{Msymbols} / \mathrm{s}$, resulting in a total network capacity of $48 \mathrm{Msymbols} / \mathrm{s}$. The main parameters for the case study are summarized in Table I.

For the coded results presented in this paper, we apply bit-interleaved coded modulation [3] using the Hiperlan II rate 1/2 constraint length 7 convolutional code with generator polynomials $g_{1}=133_{\mathrm{OCT}}$ and $g_{2}=171_{\mathrm{OCT}}$. Additionally, the Hiperlan II interleaver was used for interleaving the coded bits before modulation.

The standardized Hiperlan II channel A [13], which models typical indoor environment channels with a number of Rayleigh distributed taps and an rms delay spread of $50 \mathrm{~ns}$ is used to assess the performance. The channels are assumed block time-invariant and uncorrelated amongst the antennas in the basestation's antenna array.

\section{CLS DETECTOR}

In this section, the principles of the CLS multiuser detector proposed in this paper are discussed. This multiuser detector is applicable to flat-fading MIMO scenarios where constant modulus signals are employed. We concentrate in this section on QPSK signals. The derivation holds however for any constant modulus constellation such as M-PSK. For notational simplicity, the index $n$ is dropped in this section.

\section{A. Derivation of the CLS Detector}

In this paper we assume i.i.d. AWGN on all antennas. The derivations can, however, be generalized to the colored Gaussian noise scenario by the use of a noise pre-whitener [21]. The optimal multiuser detector for each carrier is given by the maximum likelihood (ML) detector [21], [27], [28] which minimizes the $\log$-likelihood function $\Lambda(\mathbf{x})$ given by

$$
\Lambda(\mathbf{x})=\|\mathbf{H} \cdot \mathbf{x}-\mathbf{y}\|_{2}^{2}
$$

over the set of possible constellation points $\mathbf{x}$ which are in the set $S_{M L}=\{1+j, 1-j,-1+j,-1-j\}^{U}$ for QPSK. The ML detector is thus given by

$$
\tilde{\mathbf{x}}=\arg \min _{\mathbf{x} \in S_{M L}}\|\mathbf{H} \cdot \mathbf{x}-\mathbf{y}\|_{2}^{2} .
$$

As is well known, the main disadvantage of the ML detector is its complexity: the number of points in $S_{M L}$ grows exponentially with the number of users $U$ and with the constellation order.

The main target of the CLS multiuser receiver proposed in this paper, is to reduce the detection complexity yet try to take advantage of the fact that all users apply constant modulus signals. This is achieved by expanding the minimization interval for the log-likelihood function from the optimal set $S_{M L}$ to all points which lie on the sphere $\mathbf{x}^{H} \mathbf{x}=U E_{s}$ (assuming that each user has symbol energy $E_{s}$ ). This set of points, which contains the optimal set $S_{M L}$, fulfills the constant modulus criterion. Note that this optimization interval is much tighter than the optimization interval for the ZF multiuser detector (or the decorrelator in the CDMA context), which spans the entire hyperplane $\mathbf{x}$ [28], [30]. Furthermore, its optimization interval is also tighter than the interval for the generalized MMSE detector [30] where the optimization interval is given by $\mathbf{x}^{H} \mathbf{x} \leq U E_{s}$.

The CLS receiver is thus given by

$$
\tilde{\mathbf{x}}=\arg \min _{\mathbf{x}^{H} \mathbf{x}=U E_{s}}\|\mathbf{H} \cdot \mathbf{x}-\mathbf{y}\|_{2}^{2}
$$

which constitutes a least squares problem with the additional constraint that the transmitted signals should have a constant modulus. The final estimation of the users' bits is then given by slicing $\tilde{\mathbf{x}}$.

\section{B. Mathematical Specification of the CLS Detector}

Dropping the term in the log-likelihood function which is not a function of $\mathbf{x}$, the CLS minimization problem (4) can be written as

$$
\begin{aligned}
& \min _{\mathbf{x}} \mathbf{x}^{H} \mathbf{R} \mathbf{x}-2 \operatorname{Re}\left(\mathbf{x}^{H} \mathbf{H}^{H} \mathbf{y}\right) \\
& \text { s.t. } \mathbf{x}^{H} \mathbf{x}=U E_{s}
\end{aligned}
$$

with the channel autocorrelation matrix $\mathbf{R}=\mathbf{H}^{H} \mathbf{H}$. The Lagrangian $\mathcal{L}(\mathbf{x}, \lambda)$ for this minimization problem is

$$
\mathcal{L}(\mathbf{x}, \lambda)=\mathbf{x}^{H} \mathbf{R} \mathbf{x}-2 \operatorname{Re}\left(\mathbf{x}^{H} \mathbf{H}^{H} \mathbf{y}\right)+\lambda\left(\mathbf{x}^{H} \mathbf{x}-U E_{s}\right) .
$$


Straightforward derivation gives the following solution for $\tilde{\mathbf{x}}$ :

$$
\tilde{\mathbf{x}}=\underbrace{\left(\mathbf{R}+\lambda \mathbf{I}_{U \times U}\right)^{-1} \mathbf{H}^{H}}_{\mathbf{E}_{C L S}} \mathbf{y}
$$

where $\lambda$ has to be calculated such that the constant modulus constraint is fulfilled. The solution in (7), which generates a linear equalizer $\mathbf{E}_{C L S}$, is similar to the MMSE detector [18], [27], [28] and the generalized MMSE detector [30]. When $\lambda=$ $\sigma_{n}^{2} / E_{s}$ with $\sigma_{n}^{2}$ the noise power, the CLS receiver reduces to the MMSE detector. For the generalized MMSE detector, the optimal $\lambda$ is computed iteratively over the interval $\mathbf{x}^{T} \mathbf{x} \leq U E_{s}$ [30]. Note that in (7) it is assumed implicitly that $\mathbf{R}+\lambda \mathbf{I}_{U \times U}$ is full rank, which is the case provided that $-\lambda$ does not equal one of the eigenvalues of $\mathbf{R}$. We will show later on, however, that the filters corresponding to these eigenvalues cannot be valid solutions.

In order to compute the constrained least squares filter $\mathbf{E}_{\mathrm{CLS}}$ as given by (7), the optimal value for $\lambda$ has to be found. The problem of finding this optimal $\lambda$ can be substantially simplified since the CLS receiver described by (5) constitutes a quadratically LS problem [7], [9], [11], [12] for which a similar approach as in [11, p. 580] can be followed. By using the singular value decomposition (SVD) of $\mathbf{H}=\mathbf{U} \boldsymbol{\Sigma} \mathbf{V}^{H}$, the CLS receiver can alternatively be expressed as

$\arg \min _{\overline{\mathbf{x}}^{H} \overline{\mathbf{x}}=U E_{s}}\|\boldsymbol{\Sigma} \cdot \overline{\mathbf{x}}-\overline{\mathbf{y}}\|_{2}^{2}=\arg \min _{\overline{\mathbf{x}}^{H} \overline{\mathbf{x}}=U E_{s}} \sum_{i=1}^{r}\left(\sigma_{i} \bar{x}_{i}-\bar{y}_{i}\right)^{2}$

where $\overline{\mathbf{y}}=\mathbf{U}^{H} \mathbf{y}, \overline{\mathbf{x}}=\mathbf{V}^{H} \mathbf{x}$ and $r \leq U$ is the rank of the channel matrix $\mathbf{H}$. Furthermore, $\sigma_{i}$ are the (real) singular values of $\mathbf{H}$. Using Lagrange multipliers, it is easy to show that the solution for $\overline{\mathbf{X}}$ is given by

$$
\bar{x}_{i}(\lambda)=\frac{\sigma_{i} \bar{y}_{i}}{\sigma_{i}^{2}+\lambda}, \quad i=1 \cdots r .
$$

To determine the Lagrange parameter $\lambda$, we define

$$
\phi(\lambda)=\|\overline{\mathbf{x}}(\lambda)\|_{2}^{2}=\sum_{i=1}^{r} \frac{\sigma_{i}^{2}\left|\bar{y}_{i}\right|^{2}}{\left|\sigma_{i}^{2}+\lambda\right|^{2}}
$$

and search for a solution to $\phi(\lambda)=U E_{s}$. If $\lambda$ equals $-\sigma_{j}^{2}, j=$ $1 \cdots r$, then $\phi(\lambda)=\infty$. Hence, the eigenvalues of $\mathbf{R}$ cannot be valid solutions of the CLS detector, as stated previously.

Expanding (10) results in a polynomial with order $2 r$. Solving this polynomial gives $2 r$ roots $\lambda_{j}$. In [7] and [9], it was shown that the global minimum is achieved by the maximal real root $\lambda^{*}$. Hence, only this root needs to be computed.

Since $\phi\left(-\sigma_{r}^{2}\right)=\infty$ and $\phi(\infty)=0$, it is clear that $\lambda^{*} \in I$ with $I$ the interval $\left.]-\sigma_{r}^{2}, \infty\right]$. As $\phi(\lambda)$ is monotonously decreasing on this interval, this unique real root can be found with standard root-finding techniques. In this paper, Newton's method is used because of its fast convergence. A "good" starting point $\lambda^{0}$ is found by bounding $\phi(\lambda)$ on $I$ as follows:

$$
\begin{aligned}
\phi\left(\lambda=-\sigma_{r}^{2}+\epsilon\right) & =\sum_{i=1}^{r} \frac{\sigma_{i}^{2}\left|\bar{y}_{i}\right|^{2}}{\left(\sigma_{i}^{2}-\sigma_{r}^{2}+\epsilon\right)^{2}} \\
& \geq \frac{\sigma_{r}^{2}\left|\bar{y}_{r}\right|^{2}}{\epsilon^{2}}=g(\epsilon), \quad \epsilon>0 .
\end{aligned}
$$

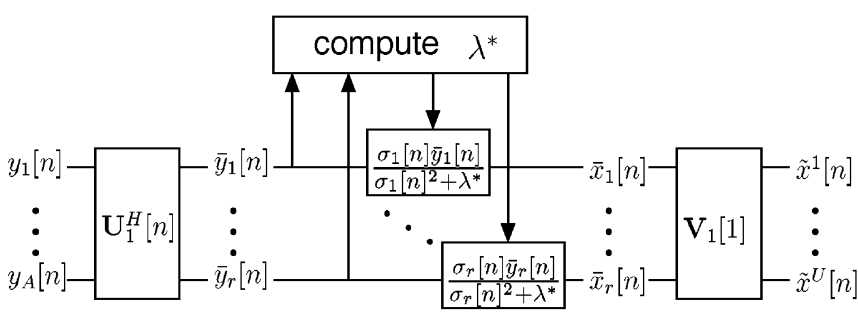

Fig. 2. Flowgraph for the CLS receiver.

Since $g(\epsilon)=U E_{s}$ for $\epsilon=\sigma_{r}\left|\bar{y}_{r}\right| / \sqrt{U E_{s}}$, the starting point given by

$$
\lambda^{0}=-\sigma_{r}^{2}+\frac{\sigma_{r}\left|\bar{y}_{r}\right|}{\sqrt{U E_{s}}}
$$

will have $\phi\left(\lambda^{0}\right) \geq U E_{s}$. Since $\phi^{\prime}(\lambda)<0$ and $\phi^{\prime \prime}(\lambda)>0$ in $I$, applying Newton's method starting from $\lambda^{0}$ results in convergence in $I$. The iterations are stopped when

$$
\left|\phi(\lambda)-U E_{s}\right| \leq T_{i t}
$$

where $T_{i t}$ is the stopping threshold which can be set to the required accuracy.

Finally, the estimation of the transmitted constant modulus vector is given by

$$
\tilde{\mathbf{x}}=\mathbf{V} \overline{\mathbf{x}}\left(\lambda^{*}\right) .
$$

Summarizing all the previous results, Fig. 2 shows a flowgraph for the CLS detector. Note that all of the computations during data processing only require the thin SVD, given by $\mathbf{H}=\mathbf{U}_{1} \boldsymbol{\Sigma}_{1} \mathbf{V}_{1}^{H}$ [11, p. 72], instead of the full SVD. If $r<U$, this reduces the processing complexity.

\section{Analysis for High SNR}

In the noiseless case, it is straightforward to show that the optimal solution is given by $\lambda=0$. Given that $\mathbf{y}=\mathbf{H} \cdot \mathbf{x}$ since no noise is present, $\phi(0)$ becomes

$$
\sum_{i=1}^{r}\left(\frac{\bar{y}_{i}}{\sigma_{i}}\right)^{2}=\|\overline{\mathbf{x}}\|_{2}^{2}=\|\mathbf{x}\|_{2}^{2}=U E_{s} .
$$

Clearly, the constant modulus criterion is fulfilled. The estimation $\tilde{\mathbf{X}}$ is then given by

$$
\tilde{\mathbf{x}}=\mathbf{R}^{-1} \mathbf{H}^{H} \mathbf{y}
$$

which corresponds to the ZF detector [28], [27], [30].

\section{Relationship With the MMSE Detector}

Since the expression for the CLS detector in (7) is so similar to the one for the MMSE detector, it seems that the estimate of the CLS detector is a scaled version of the estimate of the MMSE detector. Here, we show however that both estimates are only co-linear under very specific channel conditions. Hence, in general, the CLS detector will provide different estimates for the transmitted symbols than the MMSE detector. 
TABLE II

INITIALIZATION OPERATION COUNT PER SUBCARRIER

\begin{tabular}{rc}
\hline & Singular Value Decomposition \\
multiplications & $N_{s}\left(6 U^{3}+2 U^{2} A\right)+3 A U^{2}-\frac{U^{3}}{3}$ \\
additions & $N_{s}\left(4 U^{3}+U^{2} A\right)+3 A U^{2}-\frac{U^{3}}{3}$ \\
data transfers & $N_{s}\left(27 U^{3}+18 U^{2} A\right)+18 A U^{2}-2 U^{3}$ \\
\hline
\end{tabular}

In order for $\tilde{\mathbf{x}}_{\mathrm{CLS}}$ to be colinear with $\tilde{\mathbf{x}}_{\mathrm{MMSE}}$, it must hold that

$$
\frac{\tilde{\mathbf{x}}_{\mathrm{CLS}}}{\sqrt{U E_{s}}}=\frac{\tilde{\mathbf{x}}_{\mathrm{MMSE}}}{\left\|\tilde{\mathbf{x}}_{\mathrm{MMSE}}\right\|_{2}} .
$$

If $\left\|\tilde{\mathbf{x}}_{\mathrm{MMSE}}\right\|_{2}=\sqrt{U E_{s}}$, the MMSE detector is the solution to the CLS problem in (4) and both detectors are identical. In general, this can however not be guaranteed for the MMSE detector and it must hold that

$$
\frac{\sigma_{i}}{\sigma_{i}^{2}+\lambda}=\frac{\sqrt{U E_{s}}}{\left\|\tilde{\mathbf{x}}_{\mathrm{MMSE}}\right\|_{2}} \cdot \frac{\sigma_{i}}{\sigma_{i}^{2}+\sigma_{n}^{2} / E_{s}}, \quad \forall i=1 \cdots r
$$

However, since $\lambda$ is a constant, these relations can only hold in the degenerate case when $r=1$ or in the case when all $r$ singular values $\sigma_{i}$ are equal to a constant $\sigma$. Note that the first case occurs when there is only one user. Then, all linear detectors result in maximum ratio combining (MRC) and perform identically.

In the second case, the channel matrix possesses orthogonal columns and both the CLS detector and the MMSE detector simplify to a channel matched filter $\mathbf{H}^{H}$ followed by a scaling factor. The optimal $\lambda^{*}$ in this specific case is given by

$$
\lambda^{*}=\sigma \cdot\left(\frac{\|\mathbf{y}\|_{2}}{\sqrt{U E_{s}}}-\sigma\right) \text {. }
$$

\section{E. Complexity Analysis}

In this section, we derive the complexity of the CLS detector in terms of the number of complex operations and data transfers. The number of data transfers is an indicative number for the amount of memory/register transfers, which often dominate the implementation complexity.

A difference is made between the initialization step, when the SDMA processors are set up, and the data processing step when the actual SDMA processing is done. The former is calculated only once per frame while the latter is performed for every OFDM symbol.

1) Initialization: The CLS receiver requires the generation of the thin SVD of each channel matrix $\mathbf{H}[n]=\mathbf{U}_{1}[n] \boldsymbol{\Sigma}_{1}[n] \mathbf{V}_{1}^{H}[n]$. The approximate ${ }^{2}$ complexity for this initialization using Kogbetliantz's iterative algorithm [4], [11], [16] is shown in Table II for each subcarrier. In the derivation, the channel matrix was assumed to be full rank.

In Table II, $N_{s}$ stands for the number of "sweeps" which are executed to obtain the required accuracy. It was proven in [20] that Kogbetliantz's algorithm converges quadratically in the case of disjunct singular values. In practice, quadratic convergence is observed for all cases and only a moderate number of sweeps $N_{s}$ is needed.

${ }^{2}$ For simplicity, only the highest order terms have been retained.
TABLE III

PROCESSING OPERATION COUNT PER SUBCARRIER

\begin{tabular}{rccc}
\hline & Filter 1 & Root & Filter 2 \\
multiplications & $r A$ & $2 r+3 r N_{i t}$ & $r U$ \\
additions & $r(A-1)$ & $4 r N_{i t}$ & $U(r-1)$ \\
data transfers & $6 r A-3 r$ & $5 r+12 r N_{i t}$ & $6 r U-3 U$ \\
\hline
\end{tabular}

For the case study in Section II-C, the initialization phase requires $2.2 \mathrm{k}$ multiplications, $1.45 \mathrm{k}$ additions, and $12.5 \mathrm{k}$ data transfers per subcarrier when $N_{s}$ is assumed to be equal to 4 .

2) Processing: The processing consists of three steps. First, the received symbols $\mathbf{y}[n]$ need to be filtered with $\mathbf{U}_{1}^{H}[n]$, labeled filter 1 , to obtain $\overline{\mathbf{y}}[n]$. Then, the desired root $\lambda^{*}$ is computed using Newton's method requiring $N_{i t}$ iterations. Finally, the results are filtered with $\mathbf{V}_{1}[n]$, labeled filter 2 , to obtain $\tilde{\mathbf{x}}$. The approximate ${ }^{3}$ processing complexity for each subcarrier is shown in Table III. Note that, for Newton's method, all operations and data transfers are strictly real, reducing its complexity significantly.

Clearly, the order of the overall processing complexity is $\mathcal{O}(U A)$, which is the same as for the MMSE detector and the ZF detector [27], [28]. In the case where $r=U=A$, the 2 $A \times A$ filters in the CLS detector require twice the complexity of the $A \times A$ MMSE or ZF filters. Additionally, there is some overhead due to the root finding.

For the case study from Section II, the processing per subcarrier requires 32 multiplications, 24 additions, and 168 complex data transfers for the filtering, assuming the channel matrix to be full rank. Additionally, $8+28 N_{i t}$ real flops and $20+48 N_{i t}$ real data transfers are required for the root finding. In our simulations, where the threshold $T_{i t}$ was set to $10^{-1}$, the average number of required iterations was 3.4.

\section{Channel Estimation}

The CLS detector derived in the previous section relies on channel knowledge and therefore needs an accurate channel estimation strategy. In this section, we discuss a low-complexity MIMO channel estimator for OFDM/SDMA based on pilot symbols, in contrast with the channel estimator in [17] which uses the decoded signals as a reference. Our estimator allows multiple channel vectors $\mathbf{h}^{u}[n]$ to be estimated simultaneously on a subcarrier $n$, opposed to the pilot symbol-based estimators proposed in [15], [26] which require the users to utilize disjunct subcarriers during estimation. Furthermore, it does not rely on a specific channel model as in [6], making it more robust.

First, the pattern of these pilot symbols is discussed. Second, the estimation algorithm is analyzed.

\section{A. Pilot Pattern}

Different pilot patterns for estimating the channel matrices can be devised [6], [15], [19], [26]. Since indoor channels vary very slowly with time, we assume block time-invariant channels and rely on burst processing with periodic updating of the detection filters [26]. Hence, at the start of a frame, pilot symbols

${ }^{3}$ For simplicity, some lower order terms have been neglected in the complexity of Newton's method. 
for channel estimation are transmitted. These estimates serve to initialize the detection filters which are held constant for the rest of the frame. We have also ignored the effects of timing and frequency offsets on the system by assuming that they have been well taken care of by using timing and frequency estimation and correction.

The pilot patterns employed in this paper, are a combination of frequency interlacing (using FDMA) and spatial overlay (using CDMA). Let the number of simultaneously transmitting users on a subcarrier during estimation be denoted by $K$, where $K \leq U \leq A$. Note that the number of simultaneous users $K$ during the estimation phase is not necessarily equal to the number of users $U$ during data transmission mode. This allows a tradeoff of estimation accuracy with pilot overhead and complexity. Thus, on a certain subcarrier, a set of $K$ users are spatially overlaid allowing their combined channel matrix to be estimated simultaneously. On different subcarriers, however, different sets of users are transmitting jointly. Hence, the user sets are frequency interleaved. Finally, for each user channel estimates on each subcarrier have to be computed based on the initial estimations obtained on the pilot subcarriers.

1) Spatial Overlay: For spatial overlay of the users, we construct an orthogonal matrix $C$ with dimensions $K \times J$ such that $C C^{T}=I_{K \times K}$. To achieves this, the rows of $C$ are chosen as orthogonal Walsh-Hadamard codes c of length $J \geq K$. The estimation approach in [15], [26] corresponds to the trivial case $K=1$. The parameter $J$, which determines the estimation overhead in terms of OFDM symbols, can be varied to tradeoff estimation accuracy and pilot overhead. The minimal value of $J$ for any $K>2$, however, is given by

$$
J=4\left\lceil\frac{K}{4}\right\rceil, \quad K>2
$$

as Walsh-Hadamard matrices exist only for values $K>2$ for which $\bmod (K, 4)=0$. Here, $\lceil\cdot\rceil$ denotes rounding up to the closest integer. The pilot pattern on subcarrier $n$ is then given by

$$
\mathbf{Y}[n]=\underbrace{\left[\begin{array}{ccc}
h_{1}^{s^{1}}[n] & \cdots & h_{1}^{s^{K}}[n] \\
\vdots & \ddots & \vdots \\
h_{A}^{s^{1}[n]} & \cdots & h_{A}^{s^{K}}[n]
\end{array}\right]}_{\mathbf{H}[n]} \cdot \underbrace{\left[\begin{array}{c}
\mathbf{c}^{s^{1}}[n] \\
\vdots \\
\mathbf{c}^{s^{K}}[n]
\end{array}\right]}_{\mathbf{C}[n]}+\mathbf{N}[n]
$$

where $s^{u}$ is the $u$ th user in the user set $S$ on subcarrier $n$. The initial channel estimation $\hat{\mathbf{H}}[n]$ is obtained by right multiplying the received samples with the matrix $\mathbf{C}^{T}[n]$ as follows:

$$
\hat{\mathbf{H}}[n]=\mathbf{Y}[n] \cdot \mathbf{C}^{T}[n]=J \cdot E_{s} \cdot \mathbf{H}[n]+\underbrace{\mathbf{N}[n] \cdot \mathbf{C}^{T}[n]}_{\mathbf{N}^{\prime}[n]}
$$

where $E_{s}$ denotes the symbol energy. Assuming perfect synchronization, the spreading codes $\mathbf{c}$ with length $J$ deliver a $J$-fold increase in SNR. The estimation based on Hadamard codes has the additional advantage of not requiring multiplications.

2) Frequency Interleaving: We assume the $J$ pilot OFDM symbols to be completely filled by pilots from the different

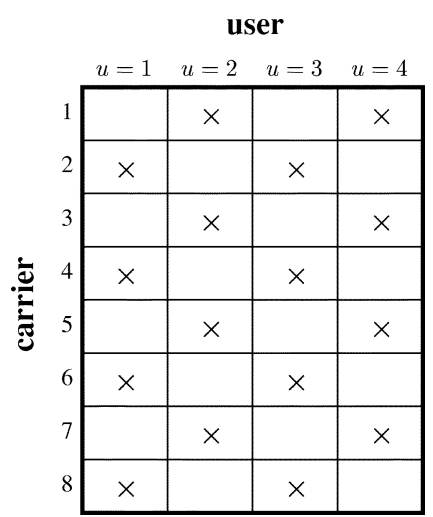

(a)

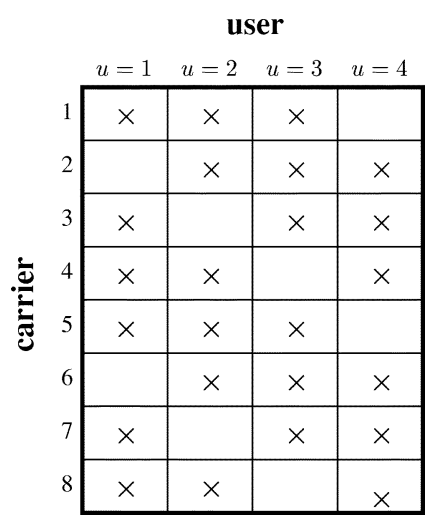

(b)
Fig. 3. Frequency interleaving pattern for $U=4$ and $N=8$.

users. The number $P$ of pilots per OFDM symbol for each user is given by

$$
P=\frac{N \cdot K}{U}
$$

where we assume for simplicity that $P$ is an integer. ${ }^{4}$ In order to maximally spread the $P$ pilots over the available subcarriers, the subcarriers assigned to user 1 are

$$
\left|\frac{N}{P}\right|,\left|2 \frac{N}{P}\right|, \ldots,\left|(P-1) \frac{N}{P}\right|, N
$$

where $|\cdot|$ here indicates rounding to the closest integer. Then, the carriers for user $u$ are found by cyclically shifting the pattern of the first user over $u-1$ subcarriers.

Exemplary frequency interleaving patterns for $U=4$ and $N=8$ are shown in Fig. 3(a) where $K=2$ and in Fig. 3(b) where $K=3$.

Finally, each user may apply a frequency code on the assigned subcarriers. However, the same code has to be used by all users in the set in order not to disturb the orthogonality within the set. This code can be optimized to different ends. For example, the code can be chosen to possess a low peak-to-average power ratio so as to minimize the nonlinearities of the receiver and the transmitter chains during channel estimation.

\section{B. Channel Estimation}

The processing in (22) delivers initial noisy estimates of the channels for each user on a set of subcarriers. For the SDMA processing, the estimation noise needs to be suppressed and channel estimates for all users on the $N_{d}$ subcarriers that actually transmit data are needed. The initial channel estimates $\hat{\mathbf{h}}_{a}^{u}[n]$ for user $u$ on antenna $a$ as given by (22) can be written as

$$
\hat{\mathbf{h}}_{a}^{u}[n]=J \cdot E_{s} \cdot \mathbf{Q}_{p}^{u} \cdot\left[\frac{\mathbf{g}_{a}^{u}[t]}{\mathbf{0}}\right]+\mathbf{n}_{a}^{u \prime}[n]
$$

where $\mathbf{Q}_{p}^{u}$ is a $P \times N$ matrix composed of $P$ rows corresponding to the pilot subcarriers of the $N \times N$ FFT matrix Q. Furthermore, $\mathbf{g}_{a}^{u}[t]$ is the time-domain channel impulse response which has a limited length $M$. We make use of a frequency smoothing and interpolation filter that tries to optimally exploit the limited

\footnotetext{
${ }^{4}$ If this is not the case, then not all users can be assigned the same number of pilots and slight pilot pattern irregularities occur.
} 
channel length in order to significantly decrease the estimation noise. To this end, (25) can be rewritten as

$$
\hat{\mathbf{h}}_{a}^{u}[n]=J \cdot E_{s} \cdot\left[\mathbf{Q}_{p h}^{u} \mid \mathbf{Q}_{p n}^{u}\right] \cdot\left[\frac{\mathbf{g}_{a}^{u}[t]}{\mathbf{0}}\right]+\mathbf{n}_{a}^{u \prime}[n]
$$

where the matrix $\mathbf{Q}_{p}^{u}$ is separated in a $P \times M$ matrix $\mathbf{Q}_{p h}^{u}$ composed of the $M$ columns corresponding to the channel and a $P \times(N-M)$ matrix $\mathbf{Q}_{p n}^{u}$ composed of the remaining columns.

In [5] and [26], the ML estimation of the channel coefficients on the $N_{d}$ data subcarriers using the initial channel estimates $\hat{\mathbf{h}}_{a}^{u}[n]$ was shown to be given by

$$
\tilde{\mathbf{h}}_{a}^{u}[n]=\frac{1}{J E_{s}} \underbrace{\mathbf{Q}_{d h}^{u} \cdot\left(\mathbf{Q}_{p h}^{u H} \cdot \mathbf{Q}_{p h}^{u}\right)^{-1} \cdot \mathbf{Q}_{p h}^{u H}}_{\mathbf{E}_{M L}^{u}} \cdot \hat{\mathbf{h}}_{a}^{u}[n]
$$

where $\mathbf{Q}_{d h}^{u}$ is a $N_{d} \times M$ matrix composed of the first $M$ elements of the $N_{d}$ rows corresponding to the data subcarriers of the FFT matrix $\mathbf{Q}$. The ML filter $\mathbf{E}_{M L}^{u}$ effectively removes part of the estimation noise and interpolates the channel estimates on the $P$ pilot carriers to obtain estimations on all data subcarriers. This ML filter corresponds to the modified least squares channel estimator first derived by van de Beek et al. in [24], which can also handle nonconstant modulus pilot symbols. Note that the same ML filter can be used for all $A$ channels $\hat{\mathbf{h}}_{a}^{u}$ corresponding to a certain user $u$.

\section{Noise Power Estimation}

For the MMSE detector, an estimate of the autocorrelation of the received signals on each subcarrier is required. In [18], where an MMSE approach closely related to MMSE-OFDM/SDMA was proposed, the correlations of the received signals are estimated directly. In our experiments, however, we estimated the noise power and used this together with the channel estimations to compute the MMSE filters directly. The noise power estimator that was used is described in the Appendix.

\section{Estimator Performance Analysis}

In this section, we study the performance of the proposed channel and noise power estimators in terms of mean square error (MSE).

1) Channel Estimation Performance: From (26) and (27), we find that

$\tilde{\mathbf{h}}_{a}^{u}[n]=\mathbf{h}_{a}^{u}[n]+\frac{1}{J \cdot E_{s}} \cdot \mathbf{Q}_{d h}^{u} \cdot\left(\mathbf{Q}_{p h}^{u H} \cdot \mathbf{Q}_{p h}^{u}\right)^{-1} \cdot \mathbf{Q}_{p h}^{u H} \cdot \mathbf{n}_{a}^{u \prime}[n]$.

Since the second term constitutes a Gaussian noise term, the proposed channel estimator is unbiased. The MSE on subcarrier $n$ for user $u$ and antenna $a$ is given by

$$
M S E_{a}^{u}[n]=\frac{\sigma_{n}^{2}}{J \cdot E_{s}} \cdot \mathbf{q}_{d h}^{u}[n] \cdot\left(\mathbf{Q}_{p h}^{u H} \cdot \mathbf{Q}_{p h}^{u}\right)^{-1} \mathbf{q}_{d h}^{u}[n]^{H}
$$

where $\mathbf{q}_{d h}^{u}[n]$ is the row of $\mathbf{Q}_{d h}^{u}$ corresponding to subcarrier $n$. The MSE differs for each subcarrier and is a function of the pilot pattern which has been used. The pilot pattern proposed in Section IV-A spreads the pilots as evenly as possible for all users and thereby aims to balance the MSE on all subcarriers.
TABLE IV

TOtal ChanNEl ESTIMATION OPERATION COUNT

\begin{tabular}{rc}
\hline multiplications & Code removal \\
additions & - \\
data transfers & $N_{d} A K(J-1)$ \\
\hline & $3 N_{d} A K(J-1)$ \\
multiplications & ML Filters \\
additions & $U A M\left(N_{d}+P\right)$ \\
data transfers & $6 U A M(P-1)+U A N_{d}(M-1)$ \\
\hline
\end{tabular}

In the special case where pilots are available on all $N$ subcarriers, (29) reduces to

$$
M S E_{a}^{u}[n]=\frac{\sigma_{n}^{2}}{J \cdot E_{s}} \cdot \frac{M}{N} .
$$

The ML filter in this case allows for a noise power reduction of $M / N$. All the noise that falls out of the first $M$ samples in the time domain corresponding to $\mathrm{g}_{a}^{u}[t]$ in (26) is rejected and only the noise which is added to $\mathrm{g}_{a}^{u}[t]$ is retained.

2) Noise Power Estimation Performance: It is easy to show that, for the noise power estimation $\tilde{\sigma}_{n}^{2}$ described in the Appendix, it holds that $\mathcal{E}\left\{\tilde{\sigma}_{n}^{2}\right\}=\sigma_{n}^{2}$, which implies that the estimator is unbiased. Furthermore, the MSE of the estimator is given by

$$
\operatorname{MSE}\left(\tilde{\sigma}_{n}^{2}\right)=\frac{\sigma_{n}^{4}}{A U(P-M)}
$$

\section{E. Estimator Complexity Analysis}

1) Channel Estimation Complexity: The total complexity of the complete channel estimation strategy, which consists of the Walsh-Hadamard code removal in (22) and the filtering with the ML filters $\mathbf{E}_{M L}^{u}$ in (27), is given in Table IV.

To reduce the complexity of the ML filtering, which clearly is the most demanding part, the filter was separated in two filters, namely $\mathbf{Q}_{d h}^{u}$ and $\left(\mathbf{Q}_{p h}^{u H} \mathbf{Q}_{p h}^{u}\right)^{-1} \mathbf{Q}_{p h}^{u H}$, which were applied consecutively. For some parameter values, however, it may be more interesting to directly apply the total filter $\mathbf{E}_{M L}^{u}$.

Note that the ML filters can be applied even more efficiently by interpreting them as the concatenation of a partial IFFT $\mathbf{Q}_{p}^{h^{H}}$, a weighting matrix $\left(\mathbf{Q}_{p}^{h^{H}} \mathbf{Q}_{p}^{h}\right)^{-1}$, and a partial FFT $\mathbf{Q}_{d}^{h}$ [5]. These (I)FFT matrices can then be pruned to remove the unnecessary branches [5], [22].

The case study of Section II requires, respectively, $2.3 \mathrm{k}$ flops and $6.9 \mathrm{k}$ data transfers for the code removal and $48.1 \mathrm{k}$ flops and $144.4 \mathrm{k}$ data transfers for the ML filters, when $K=4$.

2) Noise Power Estimation Complexity: The complexity of the noise power estimation algorithm, which consists of the filtering with $\mathbf{E}_{B L}^{u}$ in (33) and the estimation in (34), is shown in Table V.

When $K=4$, the case study of Section II, requires $49.6 \mathrm{k}$ flops and $148.9 \mathrm{k}$ data transfers, respectively. Thus, the noise estimator in this case is as complex as the channel estimator discussed in Section IV-E1. This additional complexity is however only required for the MMSE detector. The CLS detector does 
TABLE V

TOTAL NOISE POWER ESTIMATION OPERATION COUNT

\begin{tabular}{rc}
\hline & Filters \\
multiplications & $U A P(P-M)$ \\
additions & $U A(P-1)(P-M)$ \\
data transfers & $6 U A P(P-M)-3 A U(P-M)$ \\
\hline & Estimation \\
multiplications & $U A(P-M)$ \\
additions & $U A(P-M-1)$ \\
data transfers & $6 U A(P-M)-3 U A$ \\
\hline
\end{tabular}

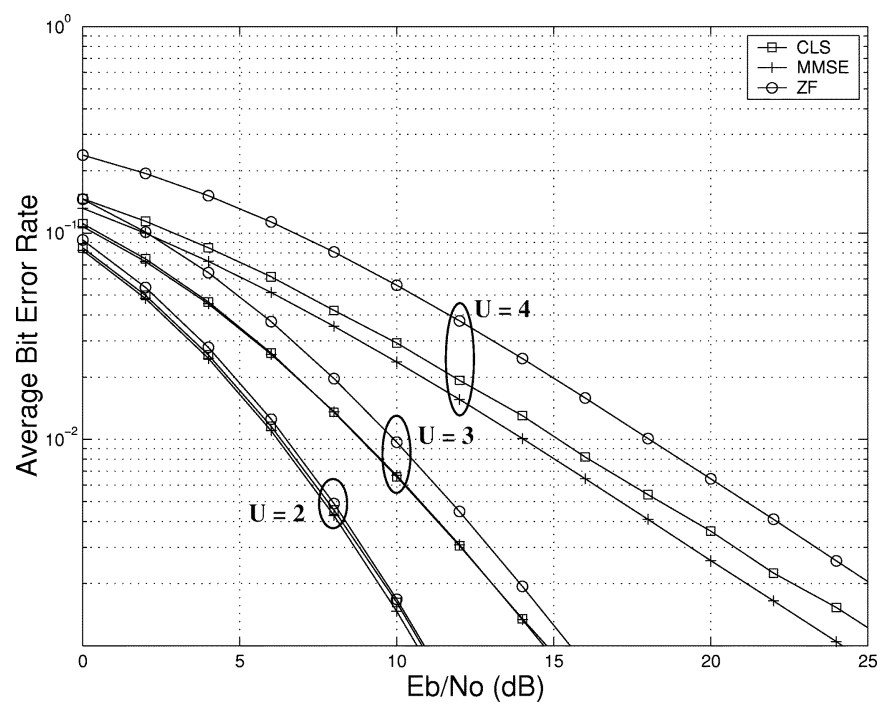

Fig. 4. Average BER versus SNR for OFDM/SDMA using QPSK with perfect channel knowledge.

not require knowledge of the noise power and hence its estimation complexity typically is substantially lower.

\section{PERformance ANALYsis}

\section{A. Results With Ideal Channel Knowledge}

Fig. 4 shows the performance of the CLS receiver for the case study defined in Section II-C when the users employ QPSK assuming ideal channel knowledge. As a reference, the performance of the ZF detector and the MMSE detector are shown. All curves are plotted for $U=2 \cdots 4$. When $U=1$, all detectors reduce to MRC [21] as stated before and behave identically. Therefore, these curves have not been plotted. For $U=4$, the CLS receiver performs within $1 \mathrm{~dB}$ of the MMSE detector and outperforms the ZF detector by $3 \mathrm{~dB}$, indicating that better detection is achieved by constraining the norm of the estimated transmit vector. For lower $U$, the differences between the detectors become smaller. For all simulations, the stopping criterion $T_{i t}$ for Newton's method was set to $10^{-1}$ which requires on the average only 3.4 iterations irrespective of the SNR. Due to the rapid convergence of Newton's method, the maximum number of iterations which was encountered in the simulations was only 7 . Hence, the worst case processing delay incurred in the CLS detection is limited. Furthermore, the processing delay could be lowered even more by limiting the maximum number of iterations at a penalty in performance. The performance for

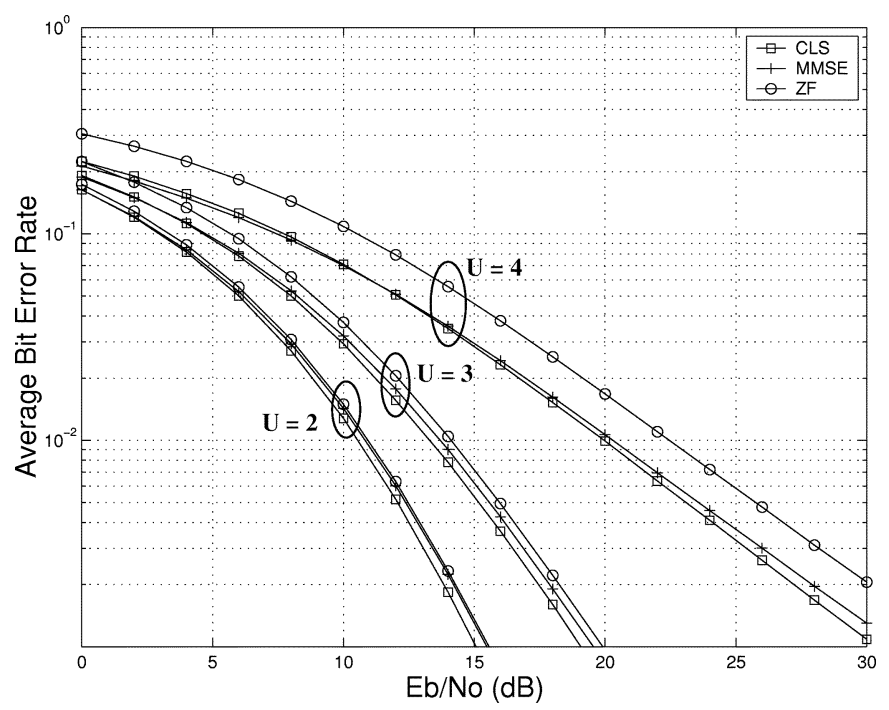

Fig. 5. Average BER versus SNR for OFDM/SDMA using 8-PSK with perfect channel knowledge.

the ZF detector was shown in [29] to correspond to the performance of QPSK signaling over a Rayleigh fading channel with $(A-U+1)$-fold diversity [21]. Note that all the detectors in Fig. 4 achieve the same order of diversity $A-U+1$.

Higher order PSK constellations lead to a higher spectral efficiency while still possessing a constant amplitude. Hence, the proposed detector can intrinsically be applied to M-PSK signals as well. Fig. 5 shows the performance with ideal channel knowledge when employing 8-PSK instead of QPSK for $U=2 \cdots 4$. For $U=4$, the CLS detector outperforms the ZF detector by $2.5 \mathrm{~dB}$ for BER $=10^{-2}$ and performs better than the MMSE for higher SNR. Here as well, the differences are smaller for lower $U$.

Finally, in Fig. 6, the performance is shown with ideal channel knowledge when employing 16-PSK for $U=2 \cdots 4$. For BER $=10^{-2}$ and $U=4$, the CLS detector now outperforms the MMSE detector by $1.5 \mathrm{~dB}$ and the ZF detector by more than $2 \mathrm{~dB}$. For all $U$, the CLS detector performs better than the MMSE detector for higher order constant modulus signals, indicating that the linear transformation corresponding to the MMSE detector does not necessarily lead to the lowest BER of all possible linear transformations. By taking the constant modulus nature of the signals as a constraint, it is possible to construct a better linear transformation via the CLS detector, which computes this transformation in a nonlinear fashion.

\section{B. Results With Channel Estimation}

All the following results focus on the case $U=A=4$ as this leads to the highest spectral efficiency.

1) Uncoded Results: For $U=4$, a spectral overlay factor $K=1$ does not fulfill the condition $P \geq M$ needed for reliable estimation. Therefore, in Fig. 7, the performance of the algorithms with both QPSK and 16-PSK is shown when the channel estimation algorithm is included for $K=2$. Clearly, the performance order of the algorithms is unchanged when compared to Fig. 4. The performance of the algorithms with perfect channel knowledge is plotted in dotted line. For QPSK, all 


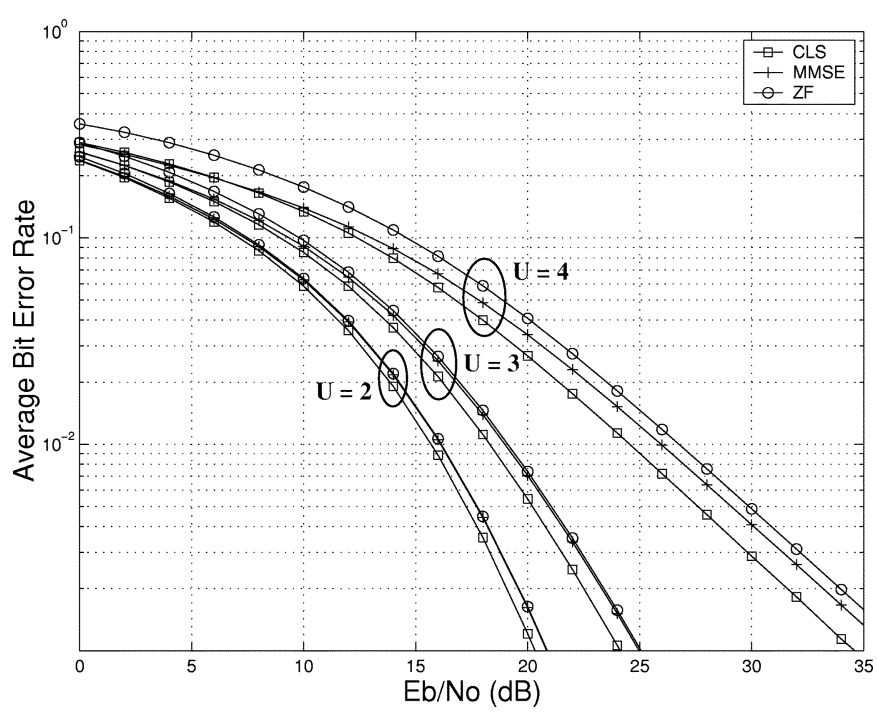

Fig. 6. Average BER versus SNR for OFDM/SDMA using 16-PSK with perfect channel knowledge.

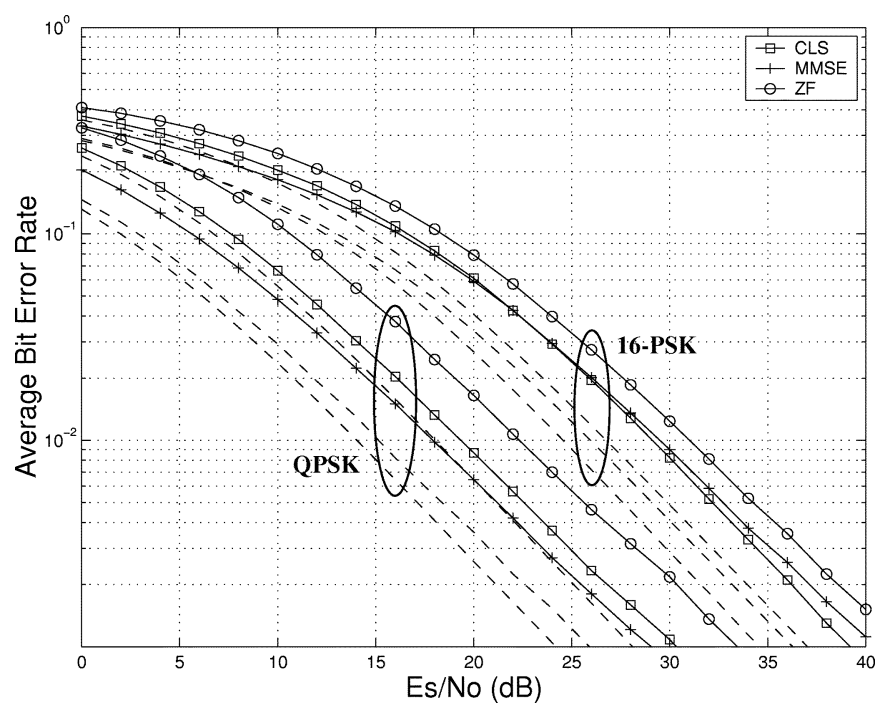

Fig. 7. Average BER versus SNR for OFDM/SDMA with channel estimation for $K=2$ and $U=4$.

algorithms lose $4 \mathrm{~dB}$ in performance compared to their ideal performance. For 16-PSK, the CLS detector has a $4.5-\mathrm{dB}$ performance penalty but, for higher $E_{s} / N_{0}$, still outperforms the MMSE detector, which has a 3.5-dB performance penalty. The expected length $\hat{M}$ of the time-domain impulse response, which is a design parameter for the smoothing filter in (27), was set equal to the length of the cyclic prefix, i.e., 16 samples. For any OFDM system, it holds that the channel lengths are expected not to exceed this length if the cyclic prefix was designed properly. The minimum code length $J$ is, in this case, equal to 2 , resulting in $P=24$ channel samples per user. Additionally, for the MMSE detector the noise power is estimated using the estimation algorithm discussed in the Appendix. In this case, $A U(P-M)=4 \times 4 \times 8=128$ noise samples are available.

In Fig. 8, the performance of the algorithms with QPSK and 16-PSK is shown when $K=3$, resulting in a code length $J=4$, $P=36$ channel samples per user, and 576 noise samples. For

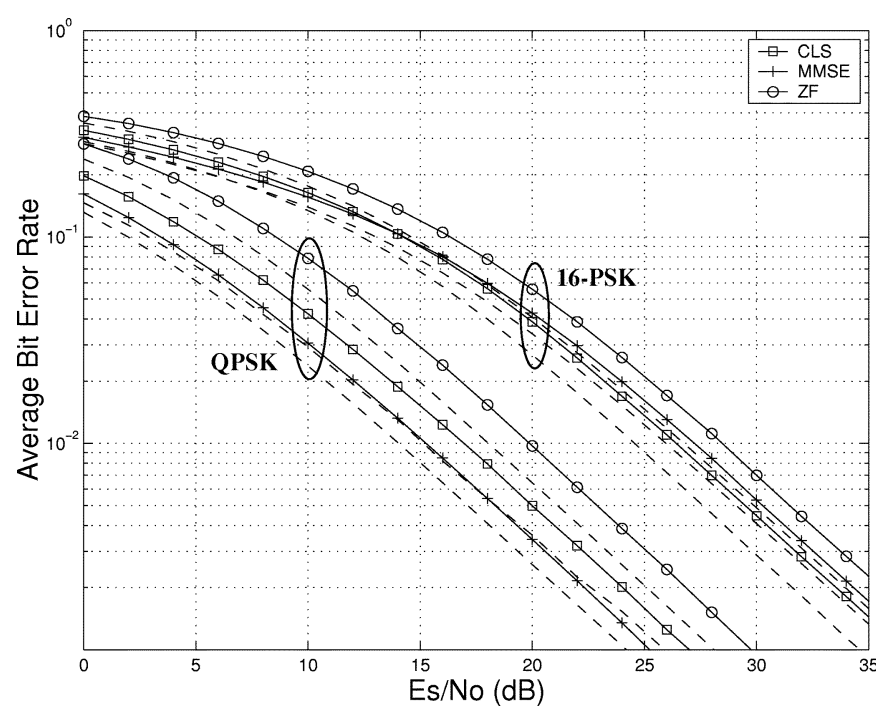

Fig. 8. Average BER versus SNR for OFDM/SDMA with channel estimation for $K=3$ and $U=4$.

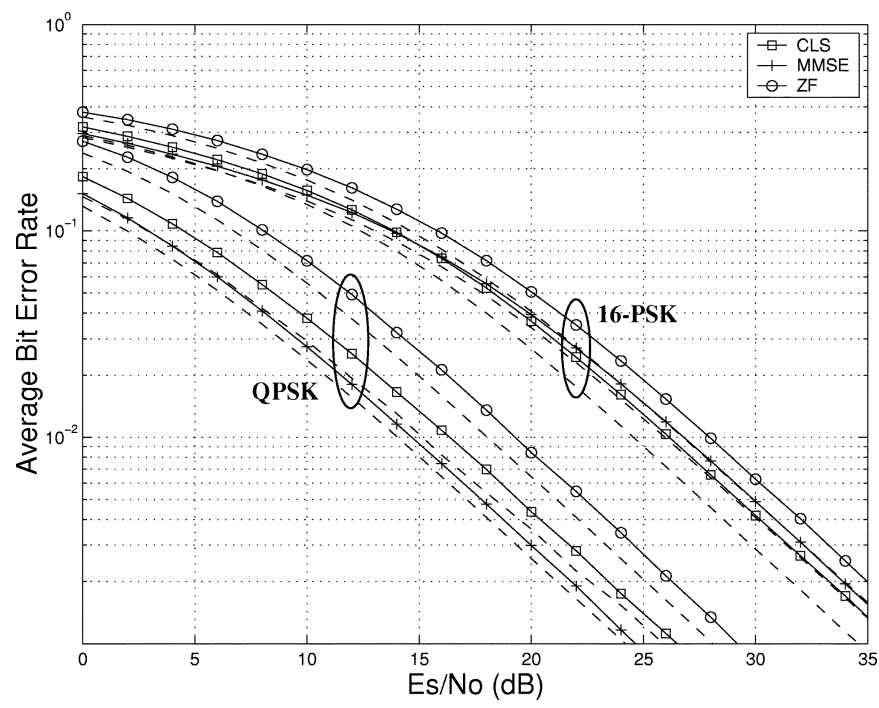

Fig. 9. Average BER versus SNR for OFDM/SDMA with channel estimation for $K=4$ and $U=4$.

QPSK, both the ZF and the CLS detector now lose $2 \mathrm{~dB}$ in performance compared to their ideal performance while the MMSE detector has a performance gap of only $1 \mathrm{~dB}$. For 16-PSK, the CLS detector degrades by $1.9 \mathrm{~dB}$, the ZF detector by $1.5 \mathrm{~dB}$ and the MMSE detector by $1.2 \mathrm{~dB}$. Setting $K=3$ is only interesting from a complexity point of view since the same pilot overhead exists as for $K=4$ but less channel samples $P$ per user are available. However, the lower values of $K$ and $P$ in this case result in a lower estimation complexity.

In Fig. 9, the performance of the algorithms with QPSK and 16-PSK is shown when $K=4$ with a code length $J=4$, $P=48$ channel samples per user, and 768 noise samples. For QPSK, both the ZF and the CLS detector now lose $1 \mathrm{~dB}$ in performance while the MMSE detector has a performance gap of $0.5 \mathrm{~dB}$. For 16-PSK, the CLS detector, which has a performance degradation of $1.5 \mathrm{~dB}$, outperfoms the MMSE detector by $0.8 \mathrm{~dB}$. 


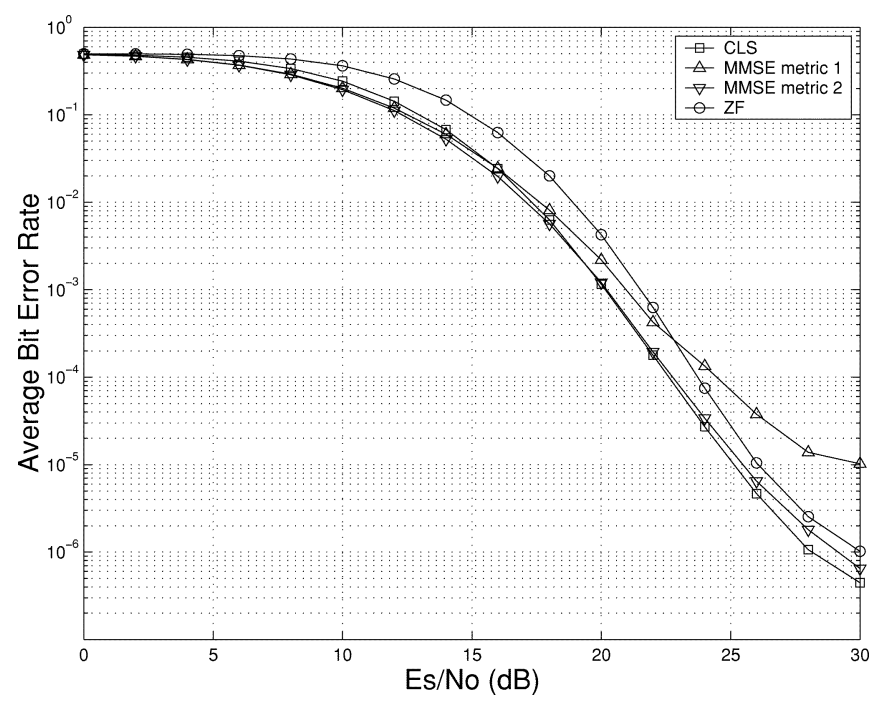

Fig. 10. Average BER versus SNR for coded OFDM/SDMA using 16-PSK for $K=4$ and $U=4$.

2) Coded Results: In Fig. 10, the performance of the three detectors is shown when channel coding is included. The curves are plotted for a fully loaded uplink $(U=4)$ using 16-PSK and channel estimation with $K=4$. Soft Viterbi decoding was performed using the simplified log likelihood metrics in [3, eq. 9]. For the MMSE detector, the results for two different metrics are plotted. The first metric only takes the noise enhancement of the MMSE filter into account for computing the equivalent noise power and neglects the residual multiuser interference. This way, knowledge of $\sigma_{n}^{2}$ is not required as this common factor in the log-likelihood metrics can be dropped. The second metric computes the equivalent noise power as the sum of the enhanced noise power and the power of the residual interference (which is thus assumed to be Gaussian distributed). The MMSE detector with the simplified metric performs worse than the ZF detector for higher SNR since the interference power has been neglected. The second metric performs substantially better but requires knowledge of the noise power $\sigma_{n}^{2}$ to compute the total equivalent noise power.

For the CLS detector, the interference of the users was neglected as no knowledge of $\sigma_{n}^{2}$ is available. Simulations showed, however, that the impact is negligible as the residual interference power is usually small compared to the noise enhancement. Hence, the CLS detector does not require knowledge of $\sigma_{n}^{2}$ for decoding, in contrast to the MMSE detector where neglecting the residual interference power results in significant performance degradation. The curves show that the coded CLS detector performs slightly better than the MMSE detector for high SNR with the best metric and outperforms the ZF detector by $1.6 \mathrm{~dB}$ for $\mathrm{BER}=10^{-2}$.

In Fig. 11, the average packet error rate (PER) after decoding is shown for the same parameters as Fig. 10. The packets consisted of 50 OFDM symbols corresponding to 9600 bits per user. Although the difference in BER was small in Fig. 10, the CLS detector performs $0.5 \mathrm{~dB}$ better in terms of PER than the MMSE detector with the second decoding metric for / hboxPER = $10^{-1}$ while for higher SNRs the gain increases further.

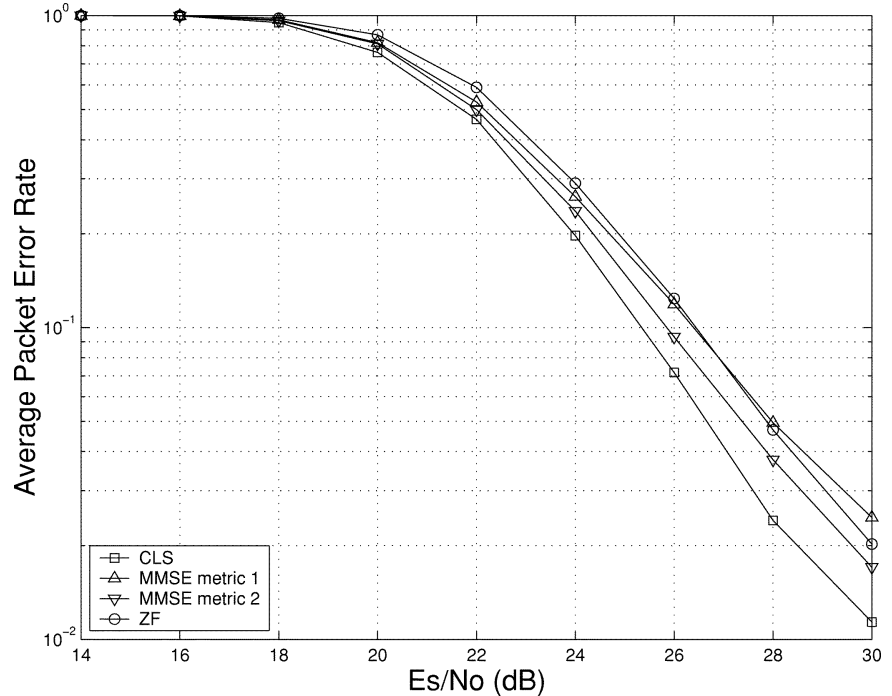

Fig. 11. Average PER versus SNR for coded OFDM/SDMA using 16-PSK for $K=4$ and $U=4$

\section{CONCLUSION}

In this paper, we proposed a new low-complexity multiuser detector and applied it to a combined OFDM/SDMA approach that combines OFDM for efficient multipath propagation mitigation with SDMA for bandwidth reuse. The proposed CLS receiver was analyzed in terms of complexity and performance. Additionally, we proposed a combined OFDM/SDMA channel and noise power estimation approach. The simulations for a Hiperlan II-based case study indicated that the CLS detector comes within $1 \mathrm{~dB}$ of the MMSE detector for the spectrally efficient case $U=A=4$ when using QPSK. For 8-PSK, the CLS detector outperforms the ZF detector by approximately 2 $\mathrm{dB}$ and performs better than the MMSE detector for higher SNR. For 16-PSK, the proposed detector is more than $2 \mathrm{~dB}$ better than the $\mathrm{ZF}$ detector and $1.5 \mathrm{~dB}$ better than the MMSE detector. Furthermore, simulations show that the performance degradation for the CLS detector due to estimation can be lowered to approximately $1 \mathrm{~dB}$ with the proposed OFDM/SDMA estimation strategy at a low pilot symbol overhead. The results including channel estimation and convolutional coding with soft Viterbi decoding when using 16-PSK indicate that the CLS detector can achieve $0.5 \mathrm{~dB}$ gain over the MMSE detector for a PER $=10^{-1}$ and more for higher SNRs.

The main advantage of the CLS detector compared to the MMSE detector is the fact that its estimation complexity is substantially lower while in our case study it performs almost as well for QPSK and better for higher order M-PSK. Therefore, the CLS detector in combination with OFDM/SDMA forms an attractive tradeoff between complexity and performance for high-speed indoor networks.

\section{APPENDIX NOISE POWER ESTIMATOR}

In this Appendix, a simple noise power estimation strategy is proposed that is necessary for the MMSE detector. The noise power spectrum is assumed to be flat although the estimation strategy can be extended to the colored noise scenario. 
Expression (26) can be rewritten as

$$
\hat{\mathbf{h}}_{a}^{u}[n]=J \cdot E_{s} \cdot \mathbf{Q}_{p h}^{u} \cdot \mathbf{g}_{a}^{u}[t]+\mathbf{n}_{a}^{u \prime}[n] .
$$

The rank of $\mathbf{Q}_{p h}^{u}$ is equal to min $(P, M)$ since this matrix can be easily shown to be full rank. For reliable channel estimation, $P$ should always exceed $M$ since the channel is assumed to have $M$ independent taps. Thus, the rank of $\mathbf{Q}_{p h}^{u}$ is always $M$ and an orthonormal $(P-M) \times P$ blocking matrix $\mathbf{E}_{\mathrm{BL}}^{u}$ can be constructed for which $\mathbf{E}_{\mathrm{BL}}^{u} \cdot \mathbf{Q}_{p h}^{u}$ is a $(P-M) \times M$ matrix with all-zero entries. The blocking matrix $\mathbf{E}_{\mathrm{BL}}^{u}$ is given by any orthonormal basis for the null space of $\mathbf{Q}_{p h}^{u}$. Then, by projecting the samples $\hat{\mathbf{h}}_{a}^{u}$ on $\mathbf{Q}_{p h}^{u \perp}$, we obtain $P-M$ noise samples $\tilde{\mathbf{n}}_{a}^{u}$

$$
\tilde{\mathbf{n}}_{a}^{u}=\mathbf{E}_{\mathrm{BL}}^{u} \cdot \hat{\mathbf{h}}_{a}^{u}[n]=\mathbf{E}_{\mathrm{BL}}^{u} \cdot \mathbf{n}_{a}^{u \prime}[n] .
$$

Note that also here the same filter can be used for all $A$ channels $\hat{\mathbf{h}}_{a}^{u}$ corresponding to a certain user $u$. The autocorrelation of the noise samples $\tilde{\mathbf{n}}_{a}^{u}$ is equal to $\sigma_{n}^{2} \cdot J \cdot E_{s} \cdot \mathbf{I}_{(P-M) \times(P-M)}$. For each combination of antenna $a$ and user $u, P-M$ noise samples are available. The ML estimation $\tilde{\sigma}_{n}^{2}$ for $\sigma_{n}^{2}$ is then given by

$$
\tilde{\sigma}_{n}^{2}=\frac{1}{A U(P-M) J E_{s}} \sum_{a=1}^{A} \sum_{u=1}^{U} \tilde{\mathbf{n}}_{a}^{u H} \cdot \tilde{\mathbf{n}}_{a}^{u} .
$$

\section{REFERENCES}

[1] T. E. Biedka, W. H. Tranter, and J. H. Reed, "Convergence analysis of the least squares constant modulus algorithm in interference cancellation applications," IEEE Trans. Commun., vol. 48, pp. 491-501, Mar. 2000.

[2] J. Bingham, "Multicarrier modulation for data transmission: An idea whose time has come," IEEE Commun. Mag., vol. 28, pp. 5-14, May 1990.

[3] G. Caire, G. Taricco, and E. Biglieri, "Bit-interleaved coded modulation," Trans. Inform. Theory, vol. 44, pp. 927-946, May 1998.

[4] J. P. Charlier, M. Vanbegin, and P. Van Dooren, "On efficient implementations of Kogbetliantz's algorithm for computing the singular value decomposition," Numer. Math., vol. 52, pp. 279-300, 1988.

[5] L. Deneire, P. Vandenameele, L. van der Perre, B. Gyselinckx, and M Engels, "A low complexity ML channel estimator for OFDM," IEEE Trans. Commun., to be published.

[6] O. Edfors, M. Sandell, J. J. van de Beek, S. K. Wilson, and P. O. Borjesson, "OFDM channel estimation by singular value decomposition," IEEE Trans. Commun., vol. 46, pp. 931-939, July 1998.

[7] G. E. Forsythe and G. H. Golub, "On the stationary values of a second degree polynomial on the unit sphere," J. Soc. Indust. Appl. Math, vol. 13, pp. $1050-1068,1965$.

[8] G. J. Foschini and M. J. Gans, "On limits of wireless communications in a fading environment when using multiple antennas," Wireless Personal Commun., vol. 6, pp. 311-335, 1998.

[9] W. Gandar, "Least squares with a quadratic constraint," Numer. Math., vol. 36, pp. 291-307, 1981.

[10] D. N. Godard, "Self-recovering equalization and carrier tracking in twodimensional data communication systems," IEEE Trans. Commun., vol. COM-28, pp. 1867-1875, Nov. 1980.

[11] G. H. Golub and C. F. Van Loan, Matrix Computations. Baltimore, MD: John Hopkins Univ. Press, 1996.

[12] G. H. Golub and U. von Matt, "Quadratically constrained least squares and quadratic problems," Numer. Math., vol. 59, pp. 561-580, 1991.

[13] ETSI Homepage [Online]. Available: http://www.etsi.org/bran.

[14] R. Jr. Johnson, P. Schniter, T. J. Endres, J. D. Behm, D. R. Brown, and R. A. Casas, "Blind equalization using the constant modulus criterion: A review," Proc. IEEE, vol. 86, pp. 1927-1950, Oct. 1998.

[15] V. K. Jones and G. G. Raleigh, "Channel estimation for wireless OFDM systems," in Globecom, Sydney, Australia, Nov. 1998, pp. 1894-1899.

[16] E. Kogbetliantz, "Solution of linear equations by diagonalization of coefficient matrices," Quart. Appl. Math., vol. 13, pp. 123-132, 1955.
[17] Y. Li, L. J. Cimini, and N. R. Sollenberger, "Robust channel estimation for OFDM systems with rapid dispersive fading channels," IEEE Trans. Commun., vol. 46, pp. 902-915, July 1998.

[18] Y. Li and N. R. Sollenberger, "Adaptive antenna arrays for OFDM systems with cochannel interference," IEEE Trans. Commun., vol. 47, no. 2, pp. 217-229, Feb. 1999

[19] R. Nilsson, O. Edfors, M. Sandell, and P. O. Borjesson, "An analysis of two-dimensional pilot symbol assisted modulation for OFDM," in Proc. ICPWC, July 1997, pp. 71-74

[20] C. Paige and P. Van Dooren, "On the quadratic convergence of Kogbetliantz's algorithm for computing the singular value decomposition," Linear Algebra Applicat., vol. 77, pp. 301-313, 1986.

[21] J. G. Proakis, Digital Communications, 3rd ed. New York: McGrawHill, 1995.

[22] H. V. Sorensen and C. S. Burrus, "Efficient computation of the DFT with only a subset of the input or output points," IEEE Trans. Signal Processing, vol. 41, p. 1184, Mar. 1993.

[23] I. E. Telatar, "Capacity of multi-antenna Gaussian channels," Eur. Trans Telecommun., vol. 10, pp. 585-595, Nov. 1999.

[24] J.-J. van de Beek, O. Edfors, M. Sandell, S. K. Wilson, and P. O. Börjesson, "On channel estimation in OFDM systems," in Proc. VTC, vol. 2, 1995, pp. 815-819.

[25] A.-J. van der Veen and A. Paulraj, "An analytical constant modulus algorithm," IEEE Trans. Signal Processing, vol. 44, pp. 1136-1155, May 1996.

[26] P. Vandenameele, L. Van der Perre, M. G. E. Engels, B. Gyselinckx, and H. J. De Man, “A combined OFDM/SDMA approach," IEEE J. Select. Areas Commun., vol. 18, pp. 2312-2321, Nov. 2000.

[27] P. Vandenameele, S. Thoen, M. Engels, and H. De Man, "A combined OFDM/SDMA approach for WLAN," in Proc. VTC Spring, Houston, TX, May 1999, pp. 1712-1716.

[28] S. Verdú, Multiuser Detection. Cambridge, U.K.: Cambridge Univ. Press, 1998.

[29] J. H. Winters, J. Salz, and R. D. Gitlin, "The impact of antenna diversity on the capacity of wireless communication systems," IEEE Trans. Commun., vol. 42, pp. 1740-1751, Feb./Mar./Apr. 1994.

[30] A. Yener, R. D. Yates, and S. Ulukus, "A nonlinear programming approach to CDMA multiuser detection," in Proc. 33th Asilomar Conf. Signals, Systems and Computers, Pacific Grove, CA, Oct. 1999, pp. 1579-1583.

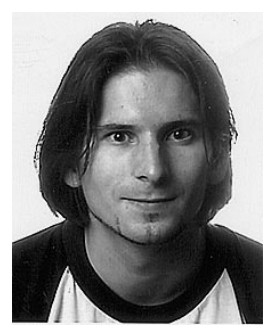

Steven Thoen (S'98) was born in Leuven, Belgium, in 1974. He received the M.Sc. degree in electrical engineering and the Ph.D. degree in communications engineering from the Katholieke Universiteit Leuven, Belgium, in 1997 and 2002, respectively.

From October 1997 until May 2002, he was with the wireless systems (WISE) group of IMEC, Leuven, Belgium, as a Ph.D. student at the Katholieke Universiteit Leuven supported by an FWO scholarship. From October 1998 to November 1998, he was a Visiting Researcher at the Information Systems Lab of Stanford University, Palo Alto, CA. In July 2002, he joined Resonext Communications, Leuven, where he is currently working on the design and implementation of advanced WLAN modems. His research interests include systems design, signal processing and digital communications systems, with particular regard to transmit optimization and MIMO transmission.

Luc Deneire (S'93-M'99) received the engineering degree in electronics from University of Liege, Belgium, in 1988, the engineering degree in telecommunications from University of Louvain-La-Neuve in 1994, and the Ph.D. degree in signal processing from Eurecom, Sophia-Antipolis, France in 1998. During this time, he was a Marie Curie Fellow grant holder.

Since late 1999, he has been a Senior Researcher at IMEC, Leuven, Belgium, the largest European independent research institute in Microelectronics, and recently joined the Signals and Systems (13S) of the University of Nice, Sophia-Antipolis, France. He is working on signal processing algorithms involved in wireless communications, specifically for third-generation mobile network, wireless LANs, and wireless personal area networks. His main interests are blind and semi-blind equalization and channel estimation, modulation theory, multiple access schemes, smart antennas, and link adaptation. He is the author of more than 40 conference and journal papers. 
Liesbet Van der Perre received the M.Sc. degree from the Katholieke Universiteit Leuven, Leuven, Belgium, in 1992 and the Ph.D. degree from the Katholieke Universiteit Leuven, Belgium, in 1997, both in electrical engineering. She performed her M.Sc. thesis research at the ENST in Paris, France.

Currently, she is the Director of the wireless program at IMEC, Leuven. Her work in the past focused on system design and digital modems for high-speed wireless communications. She was a system architect in IMEC's OFDM ASICs development and the leader of the turbo coding team. She is also a part-time Professor at the University of Antwerp, Belgium.

Marc Engels (M'96) received the engineering degree and the Ph.D. degree from the Katholieke Universiteit Leuven, Leuven, Belgium, in 1988 and 1993, respectively.

He is co-founder of LoraNet, a new company in fixed wireless access, and responsible for research and product development. The company will focus on sub-11-GHz systems that operate under non line-of-sight conditions. Technologies involved are orthogonal frequency division multiplexing (OFDM) and space division multiple access (SDMA). Prior to this, he was the Director of the wireless department at IMEC, Leuven, focused on the implementation of telecommunication systems on a chip. For these systems, he overlooked research on DSP processing, mixed-signal RF front-end, and software protocols. He was also active in design methods and tools for implementing multidisciplinary systems. Under his supervision, several systems have been realized, including a 54 Mbps WLAN terminal, a GPS-GLONASS receiver, a DECT GSM dual mode phone, and a cable modem. Previously, he performed research at the Katholieke Universiteit Leuven, Belgium, Stanford University, Stanford, CA, and the Royal Military School, Brussels, Belgium. He is a Visiting Professor of telecommunications system design at the Katholieke Universiteit Leuven and of embedded system design at the University of Lugano, Switzerland.

Dr. Engels is a member of the KVIV telecommunications society and URSI, secretary of the IEEE Benelux chapter on vehicular technology and telecommunications, and member of the board of directors of SITEL. He is an Associate Editor for the Wireless Personal Communications journal and was Associate Editor of IEEE TRANSACTIONS ON VLSI in 1999-2000.
Hugo De Man (M'81-SM'81-F'86) has been a Professor in Electrical Engineering at the Katholieke Universiteit Leuven, Leuven, Belgium, since 1976. He was a Visiting Associate Professor at the University of California at Berkeley in 1975. His early research was devoted to mixed-signal, switched capacitor, and DSP simulation tools. In 1984 he was one of the co-founders of IMEC, Leuven, which today is the largest independent semiconductor research institute in Europe with over 1100 employees. From 1984 to 1995, he was Vice President of IMEC, responsible for research in design technology for DSP and telecom applications. In 1995, he became a Senior Research Fellow of IMEC, working on strategies for education and research on design of future post-PC systems. His research at IMEC has lead to may novel tools and methods in the area of high level synthesis, hardware-software co-design and C++ based design. Many of these tools are now commercialized by spin-off companies like coware, Adelante Technologies, and Target Compilers. His work and teaching also resulted in a cluster of DSP-oriented companies in Leuven, now known as DSP Valley, where more than 1500 DSP engineers work on design tools and on telecommunications, networking, and multimedia integrated system products.

Prof. De Man received the Technical Achievement Award of the IEEE Signal Processing Society in 1999, the Phil Kaufman Award of the EDA Consortium, and the Golden Jubilee Medal of the IEEE Circuits and Systems Society. He is a member of the Royal Academy of Sciences in Belgium. 